\title{
Experimental Evaluation of Q-parameterization Controllers to a Magnetic Bearing with Imbalance
}

\author{
Non-member \\ Jun-Ho Lee(Kanazawa University) \\ Non-member Abdelfatah M. Mohamed(Assiut University) \\ Member \\ Fumio Matsumura(Kanazawa University)
}

This paper utilizes the method of Q-parameterization control to design a controller which solves the problem of imbalance in magnetic bearing systems. There are two methods to solve this problem using feedback control. The first method is to compensate for the imbalance forces by generating opposing forces on the bearing surface (imbalance compensation). The second method is to make the rotor rotate around its axis of inertia (automatic balancing);in this case no imbalance forces will be generated. The free parameter of the Q-parameterization controller is chosen such that these goals are achieved. After the introduction of a mathematical model of the magnetic bearing system, we explain the Q-parameterization controller design of the magnetic bearing system with emphasis on the rejection of sinusoidal disturbance for imbalance compensation design and the rejection of sinusoidal sensor noise for automatic balancing design. The design objectives are formulated as a linear equations in the controller free parameter Q. Finally, simulation and experimental results are presented and showed the robustness and effectiveness of the proposed controllers.

\section{INTRODUCTION}

This paper proposes a Q-parameterization control scheme for a rotating Active Magnetic Bearing(AMB) system in order to solve the problem of the imbalance. Imbalance in the rotor mass generates sinusoidal disturbance forces which cause vibration phenomena in rotating machines. Since balancing is very difficult, there is often a residual imbalance in the rotor. Moreover, the rotor sometimes becomes unbalanced while the machine is in operation. But this imbalance problem can be overcome by proper control. There are two methods to eliminate the vibration in magnetic bearing systems. The first method is to compensate for the unbalance forces by generating electromagnetic forces that cancel these forces (imbalance compensation) [1]-[5]. The second method is to make the rotor rotates around its axis of inertia (automatic balancing) [6]-[8]. In this case no unbalance forces will be generated. The Q-parameterization theory [9]-[11] characterizes the set of all stabilizing controllers of a given plant in terms of a free parameter $Q$. The controller Q-parameter is then chosen such that design specifications are achieved [7]. In this paper we utilize the Q-parameterization theory to design a controller for a magnetic bearing system to stabilize it and solve the problem of imbalance. In imbalance compensation design, the imbalance is represented by sinusoidal disturbance forces, and free parameter $Q$ of the controller is chosen such that rejection of sinusoidal disturbances is achieved. In automatic balancing design, the imbalance in the rotor is assumed as a sinusoidal noise in the measured signal. Namely the sensor measurements should indicate the motion of the principal axis of inertia plus geometrical errors due to the difference between the geometrical axis and the inertial axis. The free parameter $\mathrm{Q}$ of the controller is chosen such that rejection of sinusoidal noise is achieved; thus we can make the rotor rotates around its axis of inertia and hence achieve automatic balancing. The design objectives are formulated as a set of linear equations in the parameter $Q$. The parameter $\mathrm{Q}$ is found by simply solving this set of linear equations. In this paper, a four-axis controlled horizontal shaft magnetic bearing system is employed and the axial motion is not actively controlled.

\section{MATHEMATICAL MODEL}

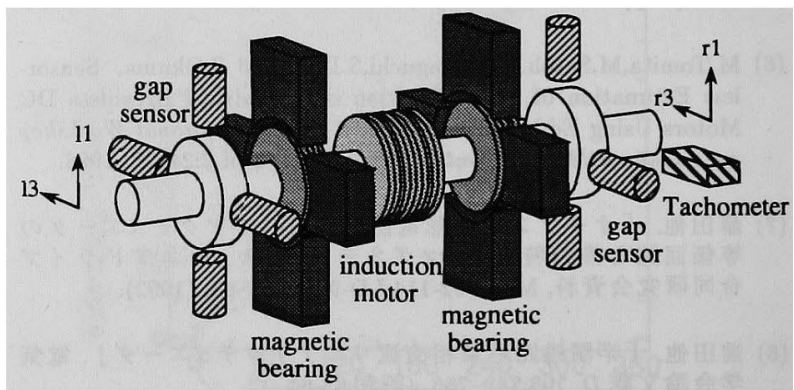

Fig. 1 Four axis magnetic bearing system

Fig. 1 shows a diagram of a four-axis controlled horizontal shaft magnetic bearing system. In our research we do not control the axial motion. The diameter of the rotor is $96 \mathrm{~mm}$ and its span is equal $660 \mathrm{~mm}$. A three-phase 
induction motor ( $1 \mathrm{~kW}$, four poles) is located at the center of the rotor. There are four pairs of electromagnets arranged radially on both sides of the rotor, and four pairs of eddy-current type gap sensors located on outside of the electromagnets. Further this system employs a tachometer in order to measure the rotational speed of the rotor. The parameters of the magnetic bearing system used in this research are given in Table 1.

Table 1: Magnetic Bearing Parameters

\begin{tabular}{|l|c|c|c|}
\hline Parameter & Symbol & Value & Unit \\
\hline Mass of the Rotor & $m$ & $1.39 \times 10^{1}$ & $\mathrm{~kg}$ \\
Moment of Inertia about X & $J_{x}$ & $1.348 \times 10^{-2}$ & $\mathrm{~kg} \cdot \mathrm{m}^{2}$ \\
Moment of Inertia about Y & $J_{y}$ & $2.326 \times 10^{-1}$ & $\mathrm{~kg} \cdot \mathrm{m}^{2}$ \\
Distance between Center of & $l_{l, r}$ & $1.30 \times 10^{-1}$ & $\mathrm{~m}$ \\
Mass and Electromagnet & & & \\
Distance between Center of & $l_{m}$ & 0 & $\mathrm{~m}$ \\
Mass and Motor & & & \\
Steady Attractive Force & $F_{l 1, r 1}$ & $9.09 \times 10$ & $\mathrm{~N}$ \\
& $F_{l 2 \sim 4, r 2 \sim 4}$ & $2.20 \times 10$ & $\mathrm{~N}$ \\
Steady Current & $I_{31, r 1}$ & $6.3 \times 10^{-1}$ & $\mathrm{~A}$ \\
& $I_{l 2 \sim 4, r 2 \sim 4}$ & $3.1 \times 10^{-1}$ & $\mathrm{~A}$ \\
Steady Gap & $W$ & $5.5 \times 10^{-4}$ & $\mathrm{~m}$ \\
Resistance & $R$ & $1.07 \times 10$ & $\Omega$ \\
Inductance & $L$ & $2.85 \times 10^{-1}$ & $\mathrm{H}$ \\
\hline
\end{tabular}

In imbalance compensation design, a mathematical model of the magnetic bearing system has been derived in reference [6], and the obtained results are as follows:

$$
\begin{aligned}
{\left[\begin{array}{c}
\dot{x}_{v} \\
\dot{x}_{h}
\end{array}\right]=} & {\left[\begin{array}{cc}
A_{v} & p A_{v h} \\
-p A_{v h} & A_{h}
\end{array}\right]\left[\begin{array}{l}
x_{v} \\
x_{h}
\end{array}\right]+\left[\begin{array}{cc}
B_{v} & 0 \\
0 & B_{h}
\end{array}\right]\left[\begin{array}{l}
u_{v} \\
u_{h}
\end{array}\right] } \\
& +p^{2}\left[\begin{array}{l}
E_{v} \\
E_{h}
\end{array}\right] W \\
& {\left[\begin{array}{l}
y_{v} \\
y_{h}
\end{array}\right]=\left[\begin{array}{cc}
C_{v} & 0 \\
0 & C_{h}
\end{array}\right]\left[\begin{array}{l}
x_{v} \\
x_{h}
\end{array}\right] }
\end{aligned}
$$

Where the states $x_{v}, x_{h}$ represent the motion of the geometrical axis and imbalance is represented by sinusoidal disturbance forces $p^{2} \mathrm{~W}$. In automatic balancing we assume that the states $x_{v}, x_{h}$ represent the motion of the inertial axis, and the imbalance is represented by a sinusoidal sensor noise $W$. In this case the sensors read the motion of the inertial axis plus the sinusoidal noise $W$ which represents the difference between the geometrical axis and the inertial axis. Then the mathematical model of the magnetic bearing system can be described by the following linear control system.

$$
\left[\begin{array}{c}
\dot{x}_{v} \\
\dot{x}_{h}
\end{array}\right]=\left[\begin{array}{cc}
A_{v} & p A_{v h} \\
-p A_{v h} & A_{h}
\end{array}\right]\left[\begin{array}{l}
x_{v} \\
x_{h}
\end{array}\right]+\left[\begin{array}{cc}
B_{v} & 0 \\
0 & B_{h}
\end{array}\right]\left[\begin{array}{l}
u_{v} \\
u_{h}
\end{array}\right]
$$

$$
\left[\begin{array}{l}
y_{v} \\
y_{h}
\end{array}\right]=\left[\begin{array}{cc}
C_{v} & 0 \\
0 & C_{h}
\end{array}\right]\left[\begin{array}{l}
x_{v} \\
x_{h}
\end{array}\right]+W
$$

where the subscripts ' $v$ ' and ' $h$ ' in the vectors and the matrices stand for the vertical motion and the horizontal motion of the magnetic bearing, respectively. In addition, the subscript ' $v h$ ' stands for the interference term between the vertical motion and the horizontal motion, and $p$ denotes the rotational speed of the rotor in $[\mathrm{rad} / \mathrm{s}]$. The vectors in equations $(1),(2),(3),(4)$ are defined as

$$
x_{v}=\left[\begin{array}{llllll}
g_{l 1} & g_{r 1} & \dot{g}_{l 1} & \dot{g}_{r 1} & i_{l 1} & i_{r 1}
\end{array}\right]^{T}
$$

$$
\begin{aligned}
& x_{h}=\left[\begin{array}{llllll}
g_{l 3} & g_{r 3} & \dot{g}_{l 3} & \dot{g}_{r 3} & i_{l 3} & i_{r 3}
\end{array}\right]^{T}, \\
& u_{v}=\left[\begin{array}{ll}
e_{l 1} & e_{r 1}
\end{array}\right]^{T}, \quad u_{h}=\left[\begin{array}{ll}
e_{l 3} & e_{r 3}
\end{array}\right]^{T}, \\
& W=\left[\begin{array}{c}
\epsilon \sin (p t+\kappa) \\
\tau \cos (p t+\lambda) \\
\epsilon \cos (p t+\kappa) \\
\tau \sin (p t+\lambda)
\end{array}\right]
\end{aligned}
$$

where

$$
\begin{aligned}
& g_{j}: \text { deviations from the steady gap lengths } \\
& \text { between the electromagnets and the rotor } \\
& i_{j}: \begin{array}{l}
\text { deviations from the steady currents of the } \\
\text { electromagnets }
\end{array} \\
& e_{j}: \begin{array}{l}
\text { deviations from the steady voltages of the } \\
\text { electromagnets }
\end{array} \\
& \epsilon, \tau, \kappa, \lambda: \text { imbalance parameters } \\
& (j=l 1, r 1, l 3, r 3 .)
\end{aligned}
$$

The subscripts ' $l$ ' and ' $r$ ' denote the left-hand side and the right-hand side of the magnetic bearing respectively, and the subscripts ' 1 ' and ' 3 ' denote the vertical directions and the horizontal directions of the rotor respectively. The different matrices in equations $(1),(2),(3),(4)$ are defined as follows.

$$
\begin{aligned}
& A_{d}:=\left[\begin{array}{ccc}
0 & I & 0 \\
A_{1}+A_{2} A_{4 d} & 0 & A_{2} A_{5 d} \\
0 & 0 & -(R / L) I
\end{array}\right] \\
& A_{v h}:=\left[\begin{array}{ccc}
0 & 0 & 0 \\
0 & A_{3} & 0 \\
0 & 0 & 0
\end{array}\right], \quad B_{d}:=\left[\begin{array}{c}
0 \\
0 \\
(1 / L) I
\end{array}\right] \text {, } \\
& C_{d}:=\left[\begin{array}{lll}
I & 0 & 0
\end{array}\right], \quad E_{d}:=\left[\begin{array}{c}
0 \\
E_{1 d} \\
0
\end{array}\right] \text {, } \\
& (d=v, h), \\
& A_{1}:=\frac{\alpha}{l_{l}+l_{r}}\left[\begin{array}{ll}
\left(l_{r}-l_{m}\right)\left(\frac{1}{m}-\frac{l_{l} l_{m}}{J_{y}}\right) & \left(l_{l}-l_{m}\right)\left(\frac{1}{m}-\frac{l_{l} l_{m}}{J_{y}}\right) \\
\left(l_{r}-l_{m}\right)\left(\frac{1}{m}+\frac{l_{r} l_{m}}{J_{y}}\right) & \left(l_{l}-l_{m}\right)\left(\frac{1}{m}+\frac{l_{r} l_{m}}{J_{y}}\right)
\end{array}\right], \\
& A_{2}::=\left[\begin{array}{cc}
-\frac{1}{m}-\frac{l_{l}^{2}}{J_{y}} & -\frac{1}{m}+\frac{l_{l} l_{r}}{J_{y}} \\
-\frac{1}{m}+\frac{l_{l} l_{r}}{J_{y}} & -\frac{1}{m}-\frac{l_{r}^{2}}{J_{y}}
\end{array}\right], \\
& A_{3}:=\frac{J_{x}}{J_{y}\left(l_{l}+l_{r}\right)}\left[\begin{array}{cc}
-l_{l} & l_{l} \\
l_{\tau} & -l_{\tau}
\end{array}\right] \text {, } \\
& A_{4 v}:=-\frac{2}{W} \operatorname{diag}\left[F_{l 1}+F_{l 2}, F_{r 1}+F_{r 2}\right] \text {, } \\
& A_{4 h}:=-\frac{2}{W} \operatorname{diag}\left[F_{l 3}+F_{l 4}, F_{r 3}+F_{r 4}\right] \text {, } \\
& A_{5 v}:=2 \operatorname{diag}\left[\frac{F_{l 1}}{I_{l 1}}+\frac{F_{l 2}}{I_{l 2}}, \frac{F_{r 1}}{I_{r 1}}+\frac{F_{r 2}}{I_{r 2}}\right] \text {, } \\
& A_{5 h}:=2 \operatorname{diag}\left[\frac{F_{l 3}}{I_{l 3}}+\frac{F_{l 4}}{I_{l 4}}, \frac{F_{r 3}}{I_{r 3}}+\frac{F_{r 4}}{I_{r 4}}\right] \text {, } \\
& E_{1 v}:=\left[\begin{array}{cccc}
-1 & l_{l}\left(1-\frac{J_{x}}{J_{y}}\right) & 0 & 0 \\
-1 & -l_{r}\left(1-\frac{J_{x}}{J_{y}}\right) & 0 & 0
\end{array}\right] \text {, } \\
& E_{1 h}:=\left[\begin{array}{cccc}
0 & 0 & 1 & l_{l}\left(1-\frac{J_{x}}{J_{y}}\right) \\
0 & 0 & 1 & -l_{r}\left(1-\frac{J_{x}}{J_{y}}\right)
\end{array}\right] \text {. }
\end{aligned}
$$


In the above equations, $\alpha$ denotes the coefficient of the force which occurs when the rotor eccentrically deviates, and hence we set $\alpha=0$.

\section{Q-PARAMETERIZATION CONTROL DESIGN}

The Q-parameterization theory [9]-[11] states that the set of all stabilizing controllers for a given plant can be characterized by a free parameter $Q$. Consider the oneparameter-control feedback system shown in Fig. 2 to control the system described by equations $(1),(2),(3),(4)$.

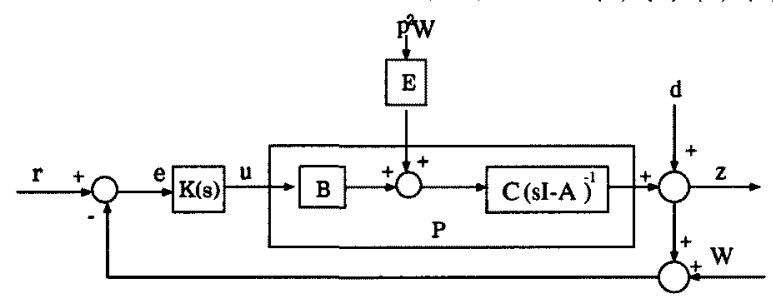

Fig. 2 One-parameter-control feedback system

Where $r$ is the reference input signal, $d$ is the output disturbance, $\mathrm{W}$ is the sensor noise, $p^{2} \mathrm{~W}$ is the disturbance force, $\mathrm{u}$ is the controller output, $\mathrm{z}$ is the plant output to be regulated, and $\mathrm{K}$ is the stabilizing controller for $\mathrm{P}(\mathrm{s})$. Note that $\mathrm{W}, p^{2} \mathrm{~W}$ may also represent model uncertainties. In order to characterize the set of all stabilizing controllers $\mathrm{K}$ for $\mathrm{P}(\mathrm{s})$ using Q-parameterization theory, first we need to construct a doubly coprime factorization $\mathrm{N}, \mathrm{M}, \widetilde{N}, \widetilde{M}, \mathrm{X}, \mathrm{Y}, \widetilde{X}, \widetilde{Y} \in \mathrm{RH} \infty$ for $\mathrm{P}(\mathrm{s})$. Let $(\mathrm{A}, \mathrm{B}, \mathrm{C})$ be system matrices of the state equations (1), (2), (3), (4), then such factorization is possible if the pairs $(A, B)$ and $(\mathrm{C}, \mathrm{A})$ are stabilizable and detectable pairs, respectively. To find $\mathrm{N}, \mathrm{M}, \tilde{N}, \widetilde{M}, \mathrm{X}, \mathrm{Y}, \tilde{X}, \tilde{Y}$, first we choose real matrices $F_{1}, F_{2}$ such that the eigenvalues of $A_{0}=\mathrm{A}-\mathrm{B} F_{1}, \widetilde{A}_{0}=\mathrm{A}-F_{2} \mathrm{C}$ have negative real parts. Then $\mathrm{N}, \mathrm{M}, \widetilde{N}, \widetilde{M}, \mathrm{X}, \mathrm{Y}, \widetilde{X}, \widetilde{Y}$ can be expressed in terms A, B, C, $A_{0}, \widetilde{A}_{0}, F_{1}, F_{2}[9] . F_{1}, F_{2}$ are obtained using the algebraic Riccati equation. With these choices, the set of all stabilizing controllers for $\mathrm{P}(\mathrm{s})$ is given by

$$
K(P)=\left\{(Y-Q \widetilde{N})^{-1}(X+Q \widetilde{M}),|Y-Q \tilde{N}| \neq 0\right\}
$$

The interpretation of the Q-parameterization control design is shown in Fig. 3 and Fig. 4. We can see from these figures that the Q-parameterization controller is an observer-based stabilizing controller with a free parameter $Q$. This free parameter can be chosen such that the design objectives are achieved.

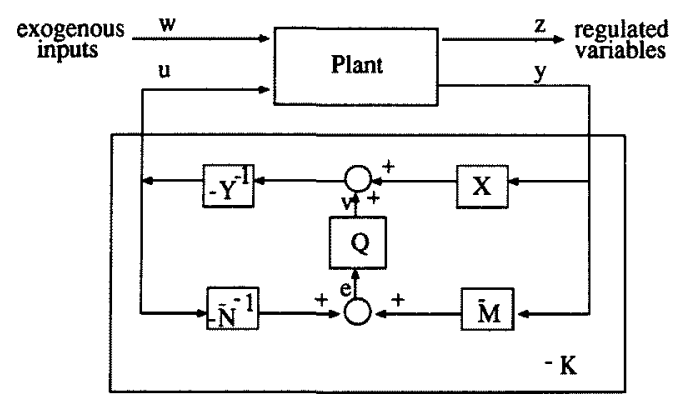

Fig. 3 Block diagram of Q-parameterization

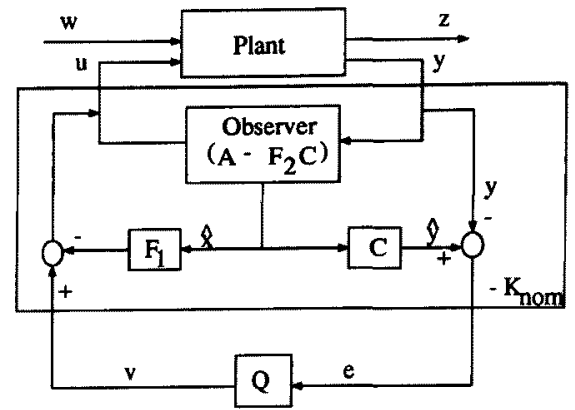

Fig. 4 Q-parameterization for estimated state feedback nominal controller

\section{CONTROLLER OBJECTIVES}

The control problem for either design (imbalance compensation or automatic balancing) can be defined as follows: Find a controller $\mathrm{K}$ such that the following requirements are satisfied:

1) The closed loop system achieves robust stability against rotor speed variation and other parameters variation, and there is fast transient response. This requirement can be satisfied if the following inequality holds.

$$
R\left(s_{i}\right)+\alpha_{s}<0
$$

where $s_{i}$ denote the closed loop poles, and $\alpha_{s}$ is a positive number chosen to ensure a certain degree of stability. In this paper $\alpha_{s}$ is chosen such that closed loop stability is achieved if the speed varies in the range $[0,22500] \mathrm{rpm}$.

2) Damping Factor: In order to prevent undesirable high frequency oscillation and to help the magnetic bearing system step through critical speeds safely, we must put a lower limit for the damping factor. To achieve this the following inequality must hold [7]

$$
\Re\left(s_{i}\right)+\beta_{d} \Im\left(s_{i}\right) \leq 0
$$

where $\beta_{d}$ is a positive constant chosen as a lower limit for the damping factor and $\Re\left(s_{i}\right)$ and $\Im\left(s_{i}\right)$ represent the real and imaginary parts of the complex number $s_{i}$.

3 ) Asymptotic rejection of low frequency disturbances. This can be achieved by making the sensitivity function small at low frequency. This requirement can be satisfied by choosing the controller Q-parameter $\mathrm{Q}$ such that

$$
W_{1}(s=0)=0
$$

where $W_{1}$ is the sensitivity transfer function i.e the transfer function from $\mathrm{d}$ to $\mathrm{z}$

$$
W_{1}=I-N(X+Q \tilde{M})
$$

4) Asymptotic rejection of sinusoidal disturbances (imbalance compensation): let $W_{2}$ be the transfer function from $p^{2} \mathrm{~W}$ to $\mathrm{z}$, then $W_{2}$ is given by

$$
W_{2}=(I-N(X+Q \tilde{M})) P_{1}(s)
$$

where $P_{1}=C(s I-A)^{-1} E$. In order to achieve this requirement $Q$ must be chosen such that the following identity hold

$$
W_{2}(s=j p)=0
$$


5) Asymptotic rejection of sinusoidal sensor noise (automatic balancing): let $W_{3}$ be the transfer function from $\mathrm{W}$ to $\mathrm{z}$, then $W_{3}$ is given by

$$
W_{3}=-N(X+Q \tilde{M})
$$

In order to achieve this requirement $\mathrm{Q}$ must be chosen such that the following identity hold

$$
W_{3}(s=j p)=0
$$

\section{CONTROLLER DESIGN}

In this section, we design the Q-parameterization controller which achieves the above requirements. At first we assume that the speed of the nominal plant is $p=0$. It means that there is no coupling between the vertical motion and horizontal motion. Therefore the plant model can be separated into vertical plant and horizontal plant.

$$
P=\left[\begin{array}{cc}
P_{v} & 0 \\
0 & P_{h}
\end{array}\right]
$$

Then, a controller will be designed for each plant. The final controller $K$ for the entire plant $P$ is constructed with the combination of these controllers as follows:

$$
K=\left[\begin{array}{cc}
K_{v} & 0 \\
0 & K_{h}
\end{array}\right]=\left[\begin{array}{cc|cc}
A_{k v} & 0 & B_{k v} & 0 \\
0 & A_{k h} & 0 & B_{k h} \\
\hline C_{k v} & 0 & D_{k v} & 0 \\
0 & C_{k h} & 0 & D_{k h}
\end{array}\right]
$$

$K_{v}$ denotes the controller for the vertical plant and $K_{h}$ denotes the controller for the horizontal plant. In order to satisfy controller requirements $(1),(2)$ we choose the matrices $F_{1}, F_{2}$ such that eigenvalues of $A_{0}, \tilde{A}_{0}$ lie in the domain D shown in Fig.5.

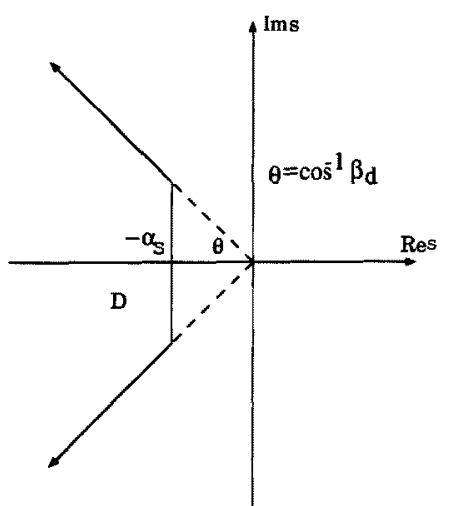

Fig.5 Generalized Region of Stability

In order to satisfy requirements $(3),(4) /(5)$ we choose the vertical controller $Q$-parameter $Q_{v}$ and horizontal controller Q-parameter $Q_{h}$ such that equations $(9),(12) /(14)$ are satisfied. From $(9)$ we have

$$
N_{x}(0)\left(X_{x}(0)+Q_{x}(0) \tilde{M}_{x}(0)\right)=I, x=v, h
$$

from (12) we have

$$
I-N_{x}(j p)\left(X_{x}(j p)+Q_{x}(j p) \tilde{M}_{x}(j p)\right)=0, x=v, h
$$

from (14) we have

$$
-N_{x}(j p)\left(X_{x}(j p)+Q_{x}(j p) \widetilde{M}_{x}(j p)\right)=0, x=v, h
$$

Equations (18),(19) are complex equations and each is in fact two equations, one for the real part and one for the imaginary part. This means that we have three equations in the unknown $Q_{x}$. Since we need to satisfy three equations, we should allow three variable coefficients. So we take the vertical controller Q-parameter $Q_{v}$ and the horizontal controller Q-parameter $Q_{h}$ in the form

$$
\begin{aligned}
& q_{v i j}(s)=\frac{a_{v i j} s^{2}+b_{v i j} s+c_{v i j}}{\left(s+p_{s 1 v}\right)\left(s+p_{s 2 v}\right)} \\
& q_{h i j}(s)=\frac{a_{h i j} s^{2}+b_{h i j} s+c_{h i j}}{\left(s+p_{s 1 h}\right)\left(s+p_{s 2 h}\right)}
\end{aligned}
$$

where $a_{v i j}, b_{v i j}, c_{v i j}, a_{h i j}, b_{h i j}, c_{h i j}(\mathrm{i}=1,2, \mathrm{j}=1,2)$ are design parameters for the vertical motion and horizontal motions, respectively, and $\left.p_{s 1 v}, p_{s 2 v}, p_{s 1 h}, p_{s 2 h}\right\rangle \alpha_{s}$ are fixed. Then we have the following linear equations. For imbalance compensation

$$
\begin{array}{ll}
A_{v 1} X_{v 1}=B_{1}, & A_{v 2} X_{v 2}=B_{2} \\
A_{h 1} X_{h 1}=B_{1}, & A_{h 2} X_{h 2}=B_{2}
\end{array}
$$

For automatic balancing

$$
\begin{array}{ll}
A_{v 1} X_{v 1}=B_{1}, & A_{v 2} X_{v 2}=B_{3} \\
A_{h 1} X_{h 1}=B_{1}, & A_{h 2} X_{h 2}=B_{3}
\end{array}
$$

where

$$
\begin{aligned}
& A_{v 1}=N_{v}(0), \quad A_{v 2}=N_{v}(j p) \\
& A_{h 1}=N_{h}(0), \quad A_{h 2}=N_{h}(j p) \\
& X_{v 1}=Q_{v}(0), \quad X_{v 2}=Q_{v}(j p) \\
& X_{h 1}=Q_{h}(0), \quad X_{h 2}=Q_{h}(j p) \\
& B_{1}=\left(\begin{array}{ll}
B_{11} & B_{12}
\end{array}\right)=\left(I-N_{x}(0) X_{x}(0)\right) \widetilde{M}_{x}(0) \\
& B_{2}=\left(\begin{array}{ll}
B_{21} & B_{22}
\end{array}\right)=\left(I-N_{x}(j p) X_{x}(j p)\right) \widetilde{M}_{x}(j p) \\
& \left.B_{3}=\left(\begin{array}{ll}
B_{31} & B_{32}
\end{array}\right)=-N_{x}(j p) X_{x}(j p)\right) \widetilde{M}_{x}(j p)
\end{aligned}
$$

where $\mathrm{x}=\mathrm{v}, \mathrm{h}$. Solving equations (22), (23) for $X_{v 1}, X_{v 2}$, $X_{h 1}, X_{h 2}$ we can easily find the design parameters $a_{v i j}$, $b_{v i j}, c_{v i j}, a_{h i j}, b_{h i j}, c_{h i j}(i=1,2, j=1,2)$ as follows:

$$
\begin{aligned}
& c_{v i j}=X_{v 1 i j} p_{s 1 v} p_{s 2 v} \\
& b_{v i j}=\Im\left\{X_{v 2 i j}\left(p_{s 1 v}+j p\right)\left(p_{s 2 v}+j p\right)\right\} / p \\
& a_{v i j}=\left(c_{v i j}-\Re\left\{X_{v 2 i j}\left(p_{s 1 v}+j p\right)\left(p_{s 2 v}+j p\right)\right\} / p^{2}(26)\right.
\end{aligned}
$$

\section{SIMULATION AND EXPERIMENTAL RE-} SULTS

We design the Q-parameterization controllers by the two methods discussed in the previous section. The controller $\mathrm{K}(\mathrm{s})$ is designed at $p=0$ and the operating speed for both imbalance compensation and automatic balancing designs is assumed to be $p=2 \pi 20[\mathrm{rad} / \mathrm{s}]$ (=1200[rpm]). The $\mu$ synthesis Toolbox [12] with Simulink were used for the controller design and simulation. 


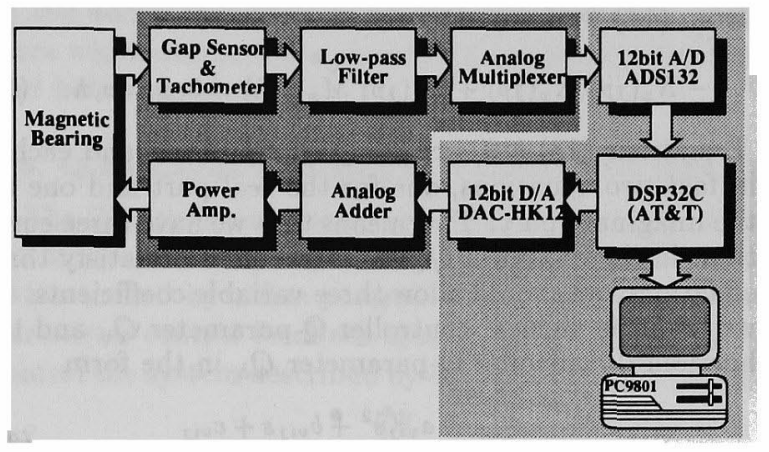

Fig. 6 Digital control system

Fig. 6 shows the digital control system for the real time implementation. The experimental machine is controlled by a digital control system that consists of a 32-bit floating point Digital Signal Processor (DSP) DSP32C(AT\&T), 12 -bit $\mathrm{A} / \mathrm{D}$ converters and 12 -bit $\mathrm{D} / \mathrm{A}$ converters. In order to make the imbalance we added a small weight $(20[\mathrm{~g}])$ to the left side of the rotor shaft. The designed continuous-time controllers are discretized via the wellknown Tustin transformation at a sampling rate of $128 \mu \mathrm{s}$.

\section{〈6.1〉 Imbalance compensation}

\section{<6.1.1) Simulation results}

We choose $p_{s 1 v}=80, p_{s 2 v}=130, p_{s 1 h}=80, p_{s 2 h}=130$, for imbalance compensation, and $F_{1 v}, F_{2 v}, F_{1 h}, F_{2 h}$ were obtained using the algebraic Riccati equation and are given by

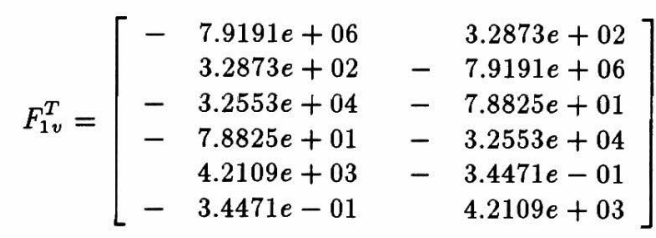

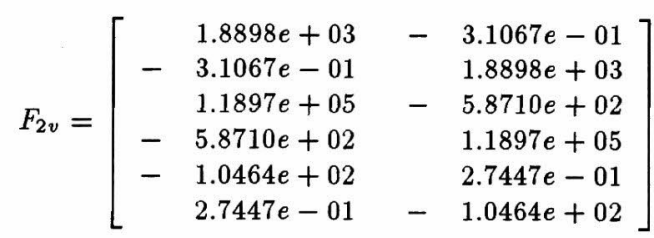

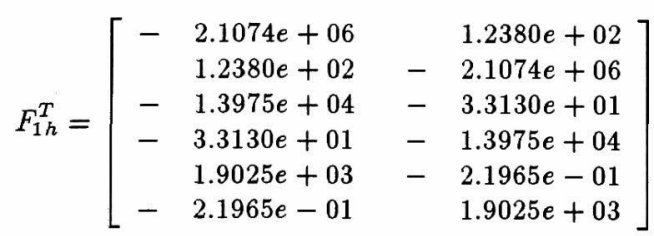

$$
\begin{aligned}
& F_{2 h}=\left[\begin{array}{rr}
1.8510 e+03 & -1.2346 e-01 \\
- & 1.2346 e-01 \\
4.6408 e+04 & -1.8510 e+03 \\
-2.2852 e+02 & \\
-1.9347 e+01 & 4.6408 e+04 \\
7.1165 e-02 & -1.1165 e-02 \\
& 1.9347 e+01
\end{array}\right]
\end{aligned}
$$

The controllers Q-parameter $Q_{v}, Q_{h}$ that can satisfy equations (17), and (18) for imbalance compensation design were found to be

$$
\begin{aligned}
& Q_{v 11}=\frac{-1.069 e+08 s^{2}-1.431 e+10 s-7.642 e+11}{s^{2}+210 s+10400} \\
& Q_{v 12}=\frac{-2.791 e+05 s^{2}-2.413 e+07 s-1.438 e+09}{s^{2}+210 s+10400} \\
& Q_{v 21}=\frac{-2.791 e+05 s^{2}-2.413 e+075-1.438 e+09}{s^{2}+210 s+10400} \\
& Q_{v 22}=\frac{-1.069 e+08 s^{2}-1.431 e+10 s-7.642 e+11}{s^{2}+210 s+10400} \\
& Q_{h 11}=\frac{-4.98 e+07 s^{2}-5.364 e+09 s-3.022 e+11}{s^{2}+210 s+10400} \\
& Q_{h 12}=\frac{-1.509 e+05 s^{2}-9.127+06 s-6.176 e+08}{s^{2}+210 s+10400} \\
& Q_{h 21}=\frac{-1.509 e+05 s^{2}-9.127+06 s-6.176 e+08}{s^{2}+210 s+10400} \\
& Q_{h 22}=\frac{-4.98 e+07 s^{2}-5.364 e+09 s-3.022 e+11}{s^{2}+210 s+10400}
\end{aligned}
$$

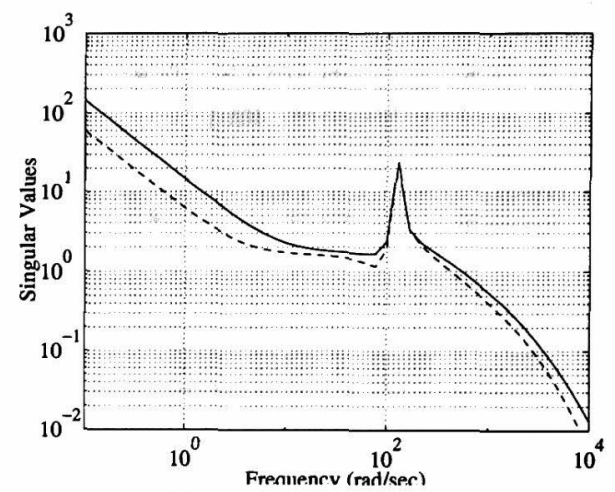

Fig. 7 Loop gain $P K$

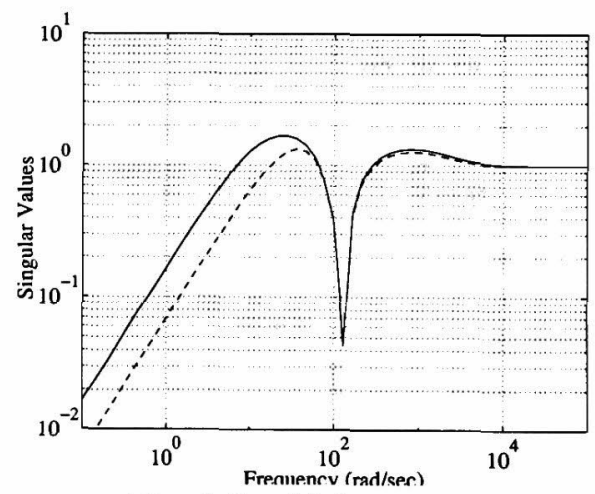

Fig. 8 Sensitivity function

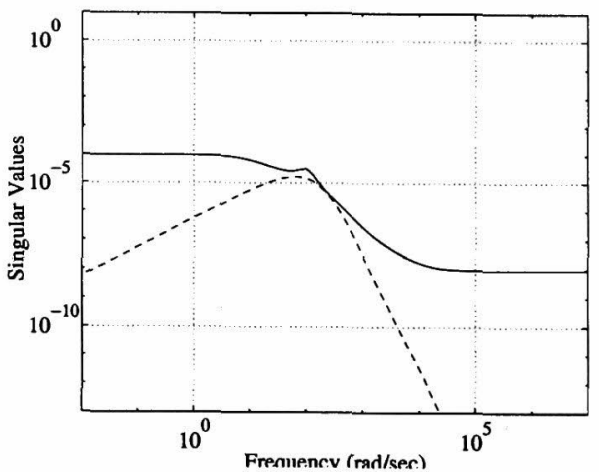

Fig. $91 / \bar{\sigma}\left(K(I+G K)^{-1}\right)(-)$ and $\bar{\sigma}\left(\Delta_{p}\right)(--)$ $p=2 \pi 375[\mathrm{rad} / \mathrm{s}]$. 
Fig. 7 shows, the singular values of the loop gain $P K$. We can see from this figure that high values of loop gain is achieved at low frequency and low values of loop gain is achieved at high frequency. Fig. 8 shows the singular values of the sensitivity function. From this figure we can see that the sensitivity function is very small at the operating speed $p=2 \pi 20[\mathrm{rad} / \mathrm{s}]$. This means good suppression of the imbalance forces is achieved. In the design of Q-parameterization controllers, we ignored the interference terms, which express the gyroscopic effect of the plant, when $p=0$. We therefore verify the robust stability of this system against changes in the rotational speed of the rotor. Let the perturbed plant $(p \neq 0)$ be denoted by $P_{p}$ and the additive perturbation $\Delta_{p}$ from $P$ is as follows:

$$
\Delta_{p}=P_{p}-P
$$

Then the robust stability is guaranteed within the the following inequality:

$$
\bar{\sigma}\left(\Delta_{p}\right)<\frac{1}{\bar{\sigma}\left(K(I-P K)^{-1}\right)}
$$

Fig. 9 shows the robust stability test for the imbalance compensation design. From this figure we can see that robust stability is achieved up to $p=2 \pi 375[\mathrm{rad} / \mathrm{s}]$ (=22500[rpm]).

\section{(6.1.2〉 Experimental results}

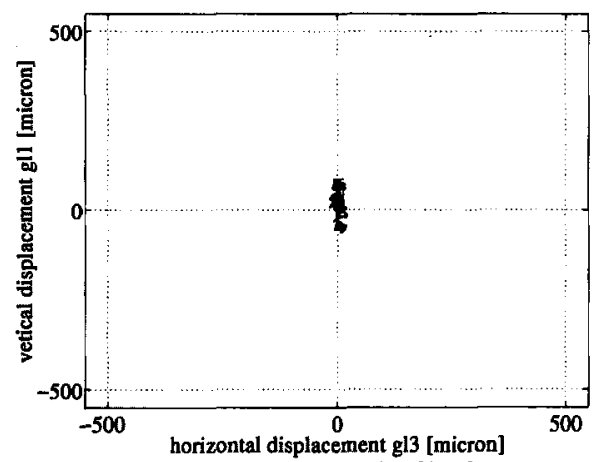

Fig. 10 Geometrical axis displacements (left side, $\mathrm{p}=1050[\mathrm{rpm}]$ )

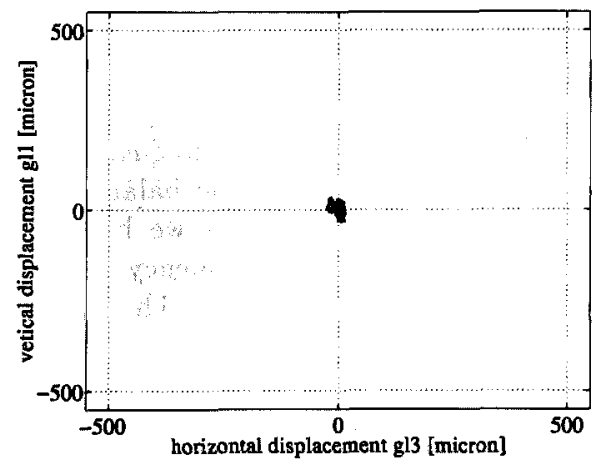

Fig. 11 Geometrical axis displacements (left side, $\mathrm{p}=1200[\mathrm{rpm}]$ )

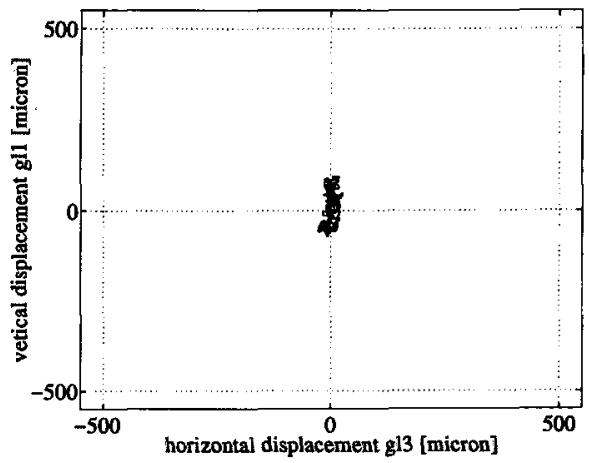

Fig. 12 Geometrical axis displacements (left side, $\mathrm{p}=1350[\mathrm{rpm}]$ )

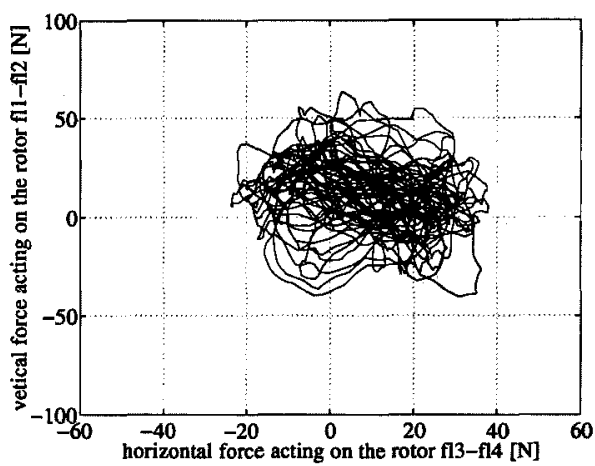

Fig. 13 Forces acting on the rotor (left side, $p=1050$ [rpm])

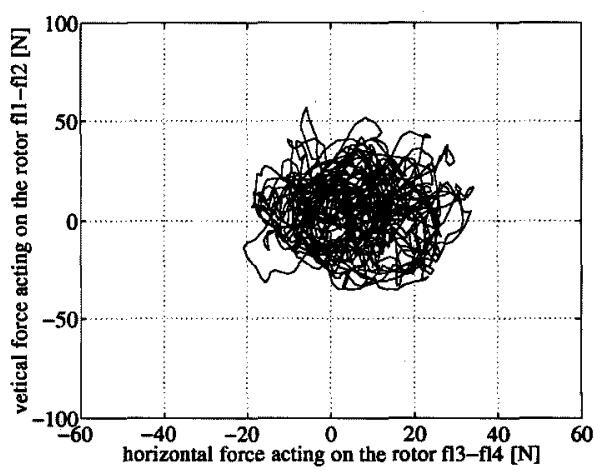

Fig. 14 Forces acting on the rotor (left side, $p=1200[\mathrm{rpm}]$ )

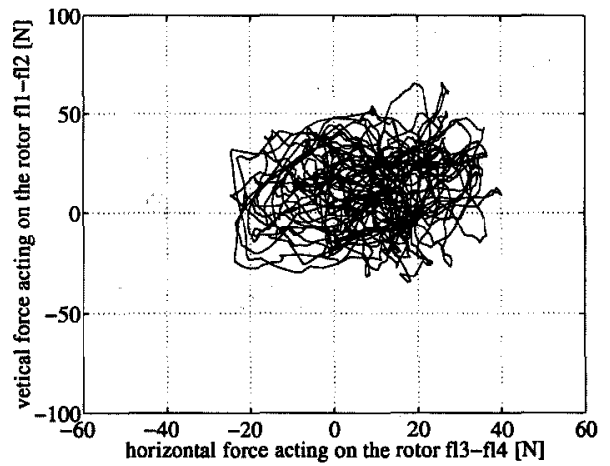

Fig. 15 Forces acting on the rotor (left side, $\mathrm{p}=1350[\mathrm{rpm}]$ ) 
Figs. $10,11,12,13,14$, and 15 show the experimental results of the geometrical axis displacements and forces acting on the rotor at different speeds for the imbalance compensation design. From these figures we can see that the geometrical axis vibration is completely eliminated at the design speed $p=2 \pi 20[\mathrm{rad} / \mathrm{s}]$ and is very small at other speeds $(p=2 \pi 17.5[\mathrm{rad} / \mathrm{s}], p=$ $2 \pi 22.5[\mathrm{rad} / \mathrm{s}])$. This means that the magnetic bearing generates sinusoidal disturbance forces which cancel the imbalance forces.

\section{(6.2) Automatic Balancing}

\section{(6.2.1) Simulation results}

We choose $p_{s 1 v}=30, p_{s 2 v}=62, p_{s 1 h}=30, p_{s 2 h}=60$, for automatic balancing, and $F_{1 v}, F_{2 v}, F_{1 h}, F_{2 h}$ were obtained using the algebraic Riccati equation and are given by.

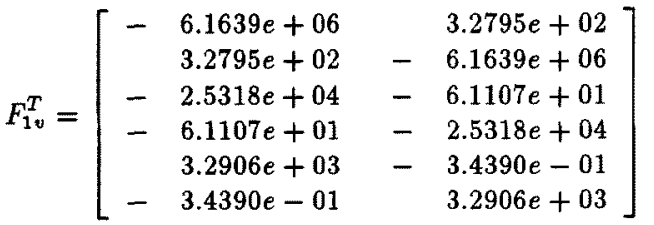

$$
\begin{aligned}
& F_{2 v}=\left[\begin{array}{rr}
1.1133 e+03 & -5.2893 e-01 \\
-5.2893 e-01 & 1.1133 e+03 \\
1.1969 e+05 & -5.8885 e+02 \\
-5.8885 e+02 & 1.1969 e+05 \\
-7.9569 e+02 & 1.4887 e+00 \\
1.4887 e+00 & -7.9569 e+02
\end{array}\right]
\end{aligned}
$$

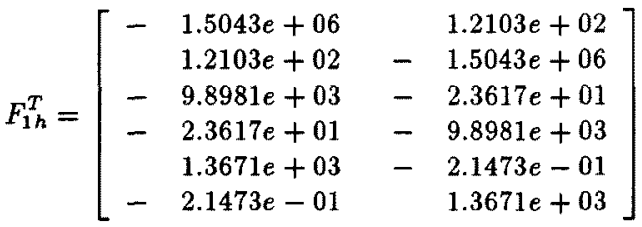

$$
\begin{aligned}
& F_{2 h}=\left[\begin{array}{rr}
5.0991 e+02 & -4.5049 e-01 \\
-4.5049 e-01 & 5.0991 e+02 \\
4.6671 e+04 & -2.2971 e+02 \\
-2.2971 e+02 & 4.6671 e+04 \\
-4.1120 e+02 & 7.8977 e-01 \\
7.8977 e-01 & -4.1120 e+02
\end{array}\right]
\end{aligned}
$$

The controllers Q-parameter $Q_{v}, Q_{h}$ that can satisfy equations (17), and (19) for automatic balancing design were found to be

$$
\begin{aligned}
& Q_{v 11}=\frac{-4.485 e+07 s^{2}-1.12 e+09 s-6.984 e+10}{s^{2}+92 s+1860} \\
& Q_{v 12}=\frac{-4.833 e+04 s^{2}+4.207 e+06 s-1.006 e+08}{s^{2}+92 s+1860} \\
& Q_{v 21}=\frac{-4.833 e+04 s^{2}+4.207 e+06 s-1.006 e+08}{s^{2}+92 s+1860} \\
& Q_{v 22}=\frac{-4.485 e+07 s^{2}-1.12 e+09 s-6.984 e+10}{s^{2}+92 s+1860} \\
& Q_{h 11}=\frac{-7.938 e+06 s^{2}+9.139 e+06 s-1.321 e+10}{s^{2}+90 s+1800} \\
& Q_{h 12}=\frac{-128.9 s^{2}+1.083 e+06 s-1.327 e+07}{s^{2}+90 s+1800} \\
& Q_{h 21}=\frac{-129.8 s^{2}+1.083 e+06 s-1.327 e+07}{s^{2}+90 s+1800} \\
& Q_{h 22}=\frac{-7.938 e+06 s^{2}+9.139 e+06 s-1.321 e+10}{s^{2}+90 s+1800}
\end{aligned}
$$

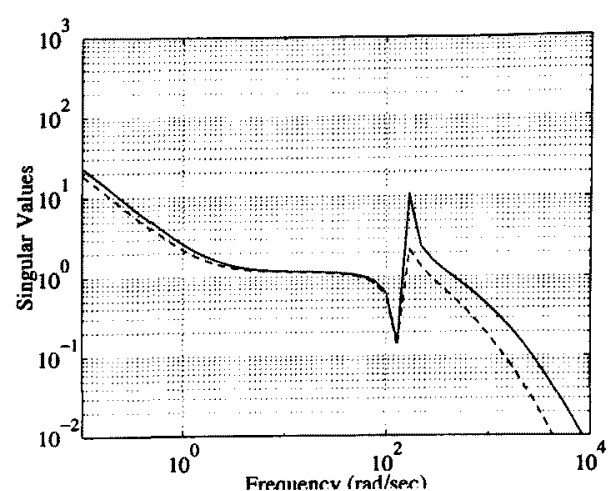

Fig. 16 Loop gain $P K$

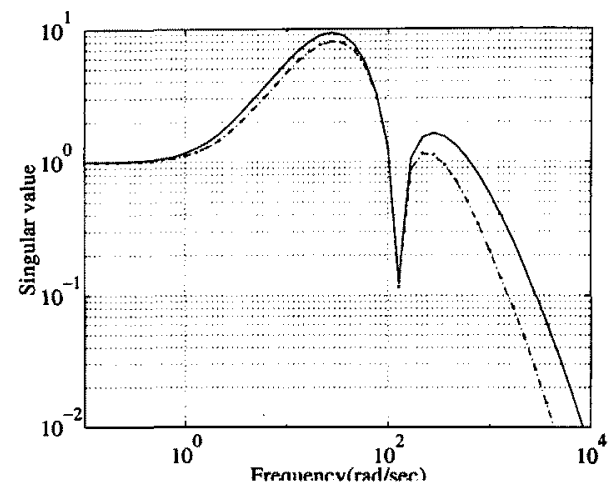

Fig. 17 Complementary sensitivity function

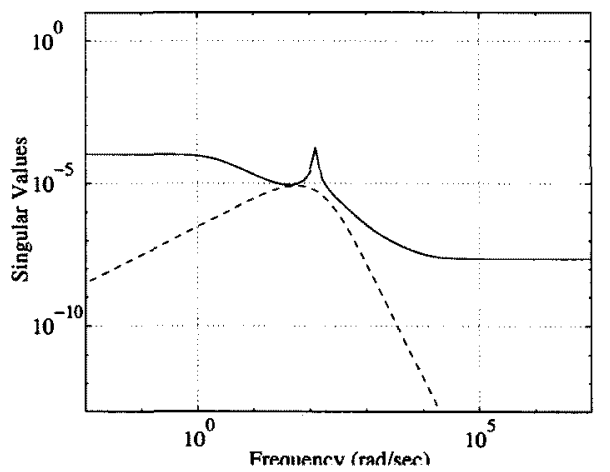

Fig. $181 / \bar{\sigma}\left(K(I+G K)^{-1}\right)(-)$ and $\bar{\sigma}\left(\Delta_{p}\right)(--)$ $p=2 \pi 200[\mathrm{rad} / \mathrm{s}]$.

Figs. 16, 17, and 18, show similar frequency response simulation results for the automatic balancing design. In this design we can also see that we have achieved high values of loop gain at low frequency and low values of loop gain at high frequency. The complementary sensitivity is very small at the design speed and this means suppression of the imbalance sinusoidal sensor noise. Fig. 18 shows the robust stability test for the automatic balancing design. From this figure we can see that robust stability is achieved up to speed $2 \pi 200[\mathrm{rad} / \mathrm{s}](=12000[\mathrm{rpm}])$. 


\section{$\langle 6.2 .2\rangle$ Experimental results}

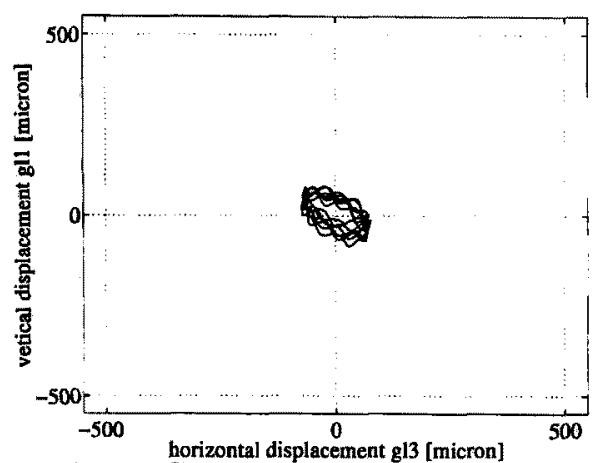

Fig. 19 Geometrical axis displacements (left side, $p=1050$ [rpm])

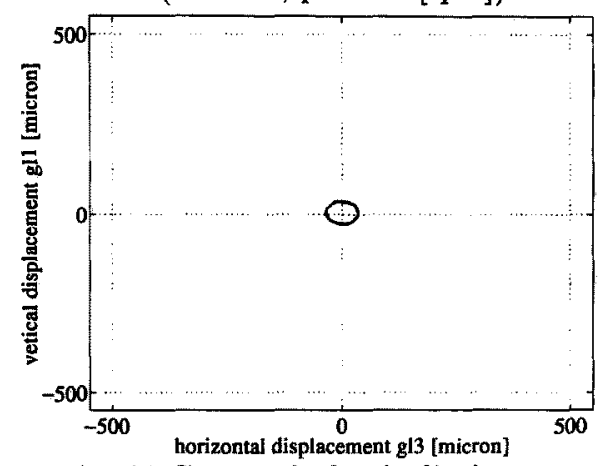

Fig. 20 Geometrical axis displacements (left side, $\mathrm{p}=1200$ [rpm])

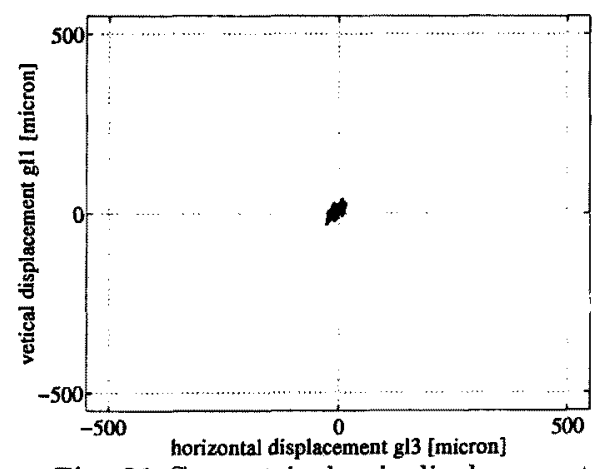

Fig. 21 Geometrical axis displacements (left side, $\mathrm{p}=1350[\mathrm{rpm}]$ )

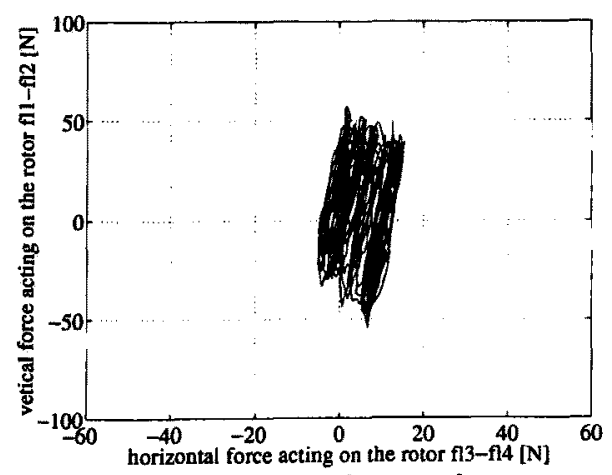

Fig. 22 Forces acting on the rotor (left side, $\mathrm{p}=1050[\mathrm{rpm}]$ )

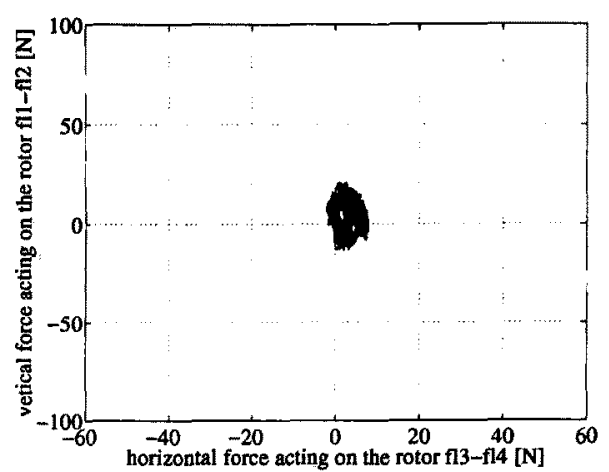

Fig. 23 Forces acting on the rotor (left side, $\mathrm{p}=1200[\mathrm{rpm}]$ )

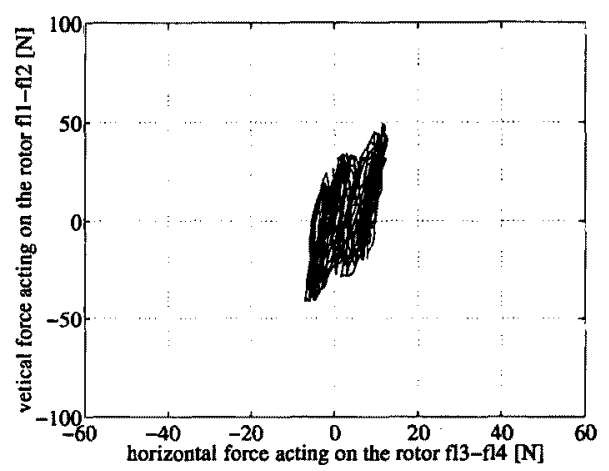

Fig. 24 Forces acting on the rotor (left side, $\mathrm{p}=1350[\mathrm{rpm}]$ )

Figs. 19, 20, 21, 22, 23, and 24 show the experimental results of the gap displacements and forces acting on the rotor at different speeds for the automatic balancing design. From these figures we can see that the magnetic forces generated by the magnetic bearing are very small at the design speed $p=2 \pi 20[\mathrm{rad} / \mathrm{s}]$, this means that the unbalance forces are very small, which indicates that the rotor rotates around its axis of inertia. In case of gap displacements, we got the most clear signal as we can see from Fig. 20. This means that while the rotor is rotating around its inertial axis, the gap sensors are sensing the geometrical axis displacements. So at the design speed we can see that almost no vibrations of the inertial axis.

\section{CONCLUSIONS}

This paper presents two methods to control the vibration caused by imbalance in the rotor of a four-axis magnetic bearing systems. To overcome the imbalance in the rotor of the magnetic bearing system, we used two different approaches. One approach is that the imbalance is modeled as sinusoidal disturbance forces. The other approach is that the imbalance is modeled as a sinusoidal sensor noise in the measured signal. The Qparameterization theory has been employed to design a controller which stabilizes the system and achieves the desired goals. The controller Q-parameter is found simply by solving a set of linear equations. The controllers that were obtained have 20 states, with 4 inputs and 4 outputs. The controller is designed at speed 
$p=0$ (nominal plant) and experimental results were obtained at three different speed $p=2 \pi 17.5[\mathrm{rad} / \mathrm{s}]$, $p=2 \pi 20[\mathrm{rad} / \mathrm{s}], p=2 \pi 22.5[\mathrm{rad} / \mathrm{s}]$ for both imbalance compensation and automatic balancing design. The results show good robustness to model uncertainties and show that the magnetic bearing systems can be used to control vibrations in rotating machinery in two different ways, by compensating for the imbalance forces (imbalance compensation) or by making the rotor rotate around its axis of inertia (automatic balancing).

(Manuscript recived Nov. 27, 1996, revised April 21, 1997)

\section{REFERENCES}

[1] F. Matsumura, T. Namerikawa, K. Hagiwara, and M. Fujita, "Application of Gain Scheduled $H_{\infty}$ Robust Controllers To A Magnetic Bearing," IEEE Transactions on Control System Technology, Vol. 4, No. 5, pp. 484-493, September. 1996.

[2] T. Mizuno and T. Higuchi, "Design of magnetic bearing controllers based on disturbance estimation," in Proc. 2nd Int. Symp Magnetic Bearing Tokyo Japan, July 12-14 1990, pp. 281-288.

[3] C. Burrows, N. Sahinkaya, A. Traxler, and G. Schweitzer, "Design and application of a magnetic bearing for vibration control and stabilization of a flexible shaft," in Proc. 1st Int. Symp Magnetic Bearing ETH Zurich, Switzerland, June 1988, pp. 159-168.

[4] C. R. Knospe, and S. Tamer, "Experiments In Robustness of Unbalance Response Controllers," in Proc.5th Int. Symp on Magnetic Bearings Kanazawa, Japan, August 28-30, 1996 pp. 131-136,

[5] S. Sivrioglu, and K. Nonami, "LMI Based Gain Scheduled $H_{\infty}$ Controller Design For AMB Systems Under Gyroscopic And Unbalance Disturbance," in Proc.5th Int. Symp on Magnetic Bearings Kanazawa, Japan, August 28-30, 1996 pp. 191196 ,

[6] F. Matsumura, M. Fujita, and K. Okawa, "Modeling and control of magnetic bearing systems achieving a rotation around the axis of inertia," Proc. 2nd Int. Symp.Magn.Bearings, Tokyo, Japan, July 12-14,1990, pp. 273-280.

[7] A. M. Mohamed, I. J. Busch-Vishniac, "Imbalance Compensation and Automation Balancing in Magnetic Bearing Systems Using the $Q$ Parameterization Theory," IEEE Trans. on Control Systems Technology, vol. 3, No. 2, pp. 202-211, June, 1995.

[8] T. Higuchi,T. Mizuno, and M. Tsukamoto, "Digital Control For Magnetic Bearings With Automatic Balancing," Proc. 2nd Int. Symp.Magn.Bearings, Tokyo, Japan July 12-14,1990, pp. 27-32.

[9] M. Vidyasagar , Control System Synthesis:A Factorization Approach. Cambridge, MA:MIT Press, 1985.
[10] J. C. Doyle, B. A. Francis, and A. R. Tannenbaum, Feed Back Control Theory. New York:Macmillan, 1992.

[11] S. Boyd, C. Barratt, Linear Controller Design: Limits Of Performance, Prentice Hall Information and Science Series New Jersey 1991.

[12] G. Balas, J. Doyle, K. Glover, A. Packard, and R. Smith, " $\mu$-Synthesis Toolbox," MuSyn Inc.,1993.

Jun-Ho,Lee (Non-member) He was born in seoul, Korea,

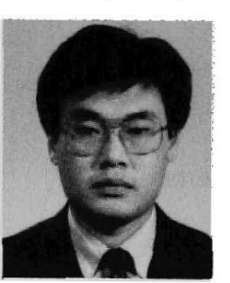
on July 3, 1964 . He received the B.E. degree, and M.E. degree in electrical engineering from KwangWoon University, Seoul, Korea, in 1987 and 1989 , respectively. $\mathrm{He}$ is currently pursuing the Ph.D. degree in electrical and computer engineering at Kanazawa University, Kanazawa, Japan. His research areas of interest are application of modern control theory,Q-parameterization robust control, $H \infty$, Loop shaping, in shaft vibration control of magnetic bearing systems and in control type magnetic levitation machines.

Abdelfatah M. Mohamed (Non-member) was born in

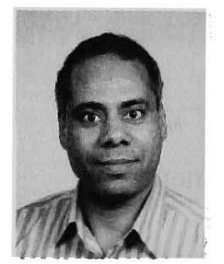
Sohag, Egypt, on September, 1953. He received the B.Sc.degree in 1976 and the M.Sc. degree in 1981 from Assiut University, Egypt and the Ph.D degree in 1990 from the University of Maryland at College Park, USA all in Electrical Engineering. Since 1990, He has been a lecturer at the Electrical Engineering Dept., Assiut University. He became an Associate Professor in 1995. From September 1991 to August 1993, He was a Postdoctoral Fellow at the Mechanical Engineering Dept., the University of Texas at Austin, USA and was on research leave from the Electrical Engineering Dept., Assiut University. Currently $\mathrm{He}$ is a visiting professor at the Electrical and Computer Engineering Dept., Kanazawa University, Japan. His research area of interest are: applications of advanced robust control such as Q-parameterization, $H_{\infty}$ optimal control, $H_{\infty}$ Loop shaping and $\mu$-Synthesis in, Magnetic bearing systems, Robotics, Industrial drives and Power systems.

Fumio Matsumura (Member) He was born in Toyama,

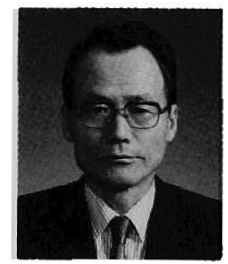
Japan, on July 27,1935 . He received the B.E. degree in electrical engineering from Kanazawa University, in 1958 and the Ph.D. degree from the University of Tokyo, in 1977. Since 1958, he has been with Kanazawa University. He is presently a Professor in the Department of Electrical and Computer Engineering. His research interests are in control type magnetic suspension systems, magnetic bearings, and linear dc motors for industrial use. He served as the general chair of the 5th International Symposium on Magnetic Bearings held in 1996. 